\title{
Other Vaccinations Given within Four Weeks
}

National Cancer Institute

\section{Source}

National Cancer Institute. Other Vaccinations Given within Four Weeks. NCI Thesaurus.

Code C102467.

Vaccines that the patient received within four weeks prior to the current vaccine administration date being reported. 